\title{
The Nearby Supernova Factory
}

\author{
W. M. Wood-Vasey *, G. Aldering, B. C. Lee, S. Loken, \\ P. Nugent, S. Perlmutter, J. Siegrist, L. Wang
}

Lawrence Berkeley National Laboratory, One Cyclotron Road, Mailstop 50R232, Berkeley, CA, 94720, USA

P. Antilogus, P. Astier, D. Hardin, R. Pain

Laboratoire de Physique Nucleaire et de Haute Energies de Paris, Paris, France

(LPNHE)

Y. Copin, G. Smadja, E. Gangler, A. Castera

Institut de Physique Nucleaire de Lyon (IPNL), Lyon, France

G. Adam, R. Bacon, J-P. Lemonnier, A. Pecontal, E. Pecontal

Centre de Recherche Astronomique de Lyon (CRAL), Lyon, France

\author{
R. Kessler \\ University of Chicago, Chicago, Illinois, USA
}

\begin{abstract}
The Nearby Supernova Factory (SNfactory) is an ambitious project to find and study in detail approximately 300 nearby Type Ia supernovae (SNe Ia) at redshifts $0.03<z<0.08$. This program will provide an exceptional data set of well-studied SNe in the nearby smooth Hubble flow that can be used as calibration for the current and future programs designed to use SNe to measure the cosmological parameters. The first key ingredient for this program is a reliable supply of Hubble-flow SNe systematically discovered in unprecedented numbers using the same techniques as those used in distant SNe searches. In 2002, 35 SNe were found using our test-bed pipeline for automated SN search and discovery. The pipeline uses images from the asteroid search conducted by the Near Earth Asteroid Tracking group at JPL. Improvements in our subtraction techniques and analysis have allowed us to increase our effective SN discovery rate to $\sim 12 \mathrm{SNe} /$ month in 2003.
\end{abstract}

Key words: supernovae, galaxies

Preprint submitted to Elsevier Science 23 October 2003 


\section{Introduction}

Type Ia supernovae (SNe Ia) have proven extremely useful as standardizable candles to explore the expansion history of the Universe (Perlmutter et al., 1997, 1998; Garnavich et al., 1998; Riess et al., 1998; Perlmutter et al., 1999). Ambitious follow-on experiments are just starting, SNLS (Pain et al., 2003), ESSENCE (Garnavich et al., 2002), or have been planned, SNAP (Aldering et al., 2002b), to extend the revolutionary result that the Universe is accelerating to precise statements about the constituents and history of the Universe. However, a key assumption underlying these experiments is that the current observed diversity in SNe Ia will be well-behaved and calibrated to allow for the desired precision measurements. This assumption can be tested in large part using nearby SNe Ia. Moreover, there is the possibility for such nearby studies to uncover new relationships that will make SNe Ia even better standard candles, much as the width-luminosity relation has brought SNe Ia to their already impressive level of standardization.

The Nearby Supernova Factory (SNfactory) project is designed to bring this improved understanding of SNe Ia (Aldering et al., 2002a; Pécontal et al., 2003; Lantz et al., 2003). Over the course of three years, it will study $300 \mathrm{SNe}$ Ia in the nearby smooth Hubble flow. These SNe will be observed with a dedicated instrument, the SuperNova Integral Field Spectrograph (SNIFS), which is currently in the final stages of construction. SNIFS will provide simultaneous spectrophotometric coverage of both the SN and the host galaxy at 3-5 day intervals during the rise and fall of each SN. This unprecedented dataset will provide a wealth of information on SNe Ia and allow for an improved calibration of SNe Ia for use in cosmology.

\section{The Supernova Search Dataset}

The SNfactory searches for SNe using wide-field images obtained in collaboration with the Near Earth Asteroid Tracking (NEAT) group (Pravdo et al., 1999) at the Jet Propulsion Laboratory (JPL). In their quest for asteroids, the NEAT group observes hundreds of fields each night by taking three images of each field, spaced fifteen to thirty minutes apart, and searching for objects that move by more than a couple of arcseconds over this period. The SNfactory uses this temporal spacing to eliminate asteroids and reduce cosmic-ray contamination. The NEAT observing program covers the observable sky from

\footnotetext{
* WMWV was supported in part by an NSF Graduate Research Fellowship. Email address: wmwood-vasey@lbl.gov (W. M. Wood-Vasey).

URL: http://snfactory.lbl.gov (W. M. Wood-Vasey).
} 
-40 to +40 degrees in declination every one to two weeks.

Since 2001, the Palomar 1.2-m. Oschin telescope has been used by the SNfactoryNEAT collaboration. The NEAT group outfitted this telescope with an automated system for control and observations and added a 3 -chip, $3 \square^{\circ}$ field-ofview CCD camera (NEAT12GEN2) at the spherical focal plane of this Schmidt reflector. We spent 2001 designing, testing, and verifying the search pipeline with the large data stream from this telescope. Full search operations began in the fall of 2002 and continued through April of 2003 when the NEAT12GEN2 camera was replaced by the Yale QUEST group (The QUEST Collaboration, 2003) with a 112-chip, $9 \square^{\circ}$ field-of-view camera (QUESTII) capable of both drift-scan and point-and-track observations. QUESTII became operational in August 2003 and is now providing data for the SNfactory search. The NEAT group also uses the Haleakala 1.2-m MSSS telescope, but as this telescope has a smaller field of view and poor image quality, the SNfactory has focused its search efforts on the images from the Palomar Oschin telescope.

\section{$3 \quad$ Data Processing}

The SNfactory, in collaboration with the High Performance Wireless Research and Education Network (Braun, 2003), has established a high-speed, 6 Megabyte-per-second (MBps) radio internet link to the San Diego Supercomputer Center (SDSC) from the Palomar observatory. Images are transmitted from the telescope and stored at the National Energy Research Supercomputing Center (NERSC) High Performance Storage System (HPSS). The bandwidth from SDSC to NERSC allows for near real-time transfer of 20-50 GB per night. From HPSS the data are transferred to the 200-node NERSC Parallel Distributed Systems Facility (PDSF) and are submitted for simultaneous processing on the PDSF cluster in groups based on the dark frame taken closest in time. The processed images are then registered with our image database, renamed to match our canonical name format, and saved to HPSS.

The SNfactory searches these data for SNe using image subtraction. The computers use a sophisticated suite of image tools, but there remains a significant, although ever-decreasing, amount of human interpretation needed to discriminate the good SN candidates from the bad. The same software used by the SNfactory to search for nearby $(\mathrm{z} \leq 0.1)$ SNe is used by the Supernova Cosmology Project (SCP) to search for distant SNe. Our subtraction software begins by spatially registering all of the images of a given field to a common reference system. The images to be used as a reference are stacked into a single coadded image, while the images to be searched are coadded into two separate images to allow for later checks for asteroids and cosmic rays. To account for differences in the effective point-spread-function from variations in atmospheric and 
telescope conditions, we calculate a convolution kernel to match each coadded image to the worst-seeing coadded image of the set. An automated scanning program looks for objects in the convolution-matched, subtracted image and applies a variety of selection criteria to eliminate cosmic rays, asteroids, and subtraction and detector artifacts. This program compiles a list of interesting candidates to be looked at in more detail by a human scanner. Every day, human scanners consult the list of interesting candidates and decide whether or not a computer-flagged candidate appears to be a real, variable object. Once we have a promising candidate, we submit it to the target list for the next night of observation with NEAT. After obtaining confirmation images of a candidate that reveal it to exhibit the appropriate behavior for a SN, we announce the apparent SN in the IAU Circulars. A confirmation spectrum is desirable but currently not always possible. When the SNIFS instrument is installed on the Hawaii 2.2-m telescope, it will be automatically scheduled to confirm and follow SNe. Spectra will be taken of each SN at 3-5 day intervals over a span of roughly 60 days.

\section{Results}

Eighty-three SNe have been found using the techniques described above and have been accepted by the International Astronomical Union. Fig. 1 shows the $35 \mathrm{SNe}$ we discovered in 2002. In the first five months of 2003, we found and reported an additional 48 SNe. In addition, our search identified another 17 SNe that had already been reported by other groups. We are currently running with a very conservative set of selection criteria and need human eyes to scan $\sim 5 \%$ of the successful subtractions for each night. However, many of the SNe discovered in 2003 could have been found using very restrictive criteria that would have required human scanning for $<1 \%$ of the subtractions. To further reduce our scanning burden, efforts are ongoing to understand the parameter space of our candidate scores to uniquely identify the SNe.

In order to understand our search results, we have developed a simple search simulator. We use a V-band lightcurve template from Goldhaber et al. (2001) to model the rise and fall of SNe Ia. ${ }^{1}$ The simulator calculates the amount of time a supernova would be visible at a given redshift assuming a normal $($ stretch $=1)$ SN Ia. We considered a redshift range from $0<z<0.2$, limiting unfiltered magnitude of 19.5, and several different repeat coverage cadences for our simulations. We included the typical NEAT sky coverage rate, $S_{D}=500 \square^{\circ} /$ night, and assumed $S_{O}=10,000 \square^{\circ}$ of usable sky a night. There is a maximum effective cadence, $C_{\max }$, beyond which one is just idling the

$\overline{1}$ We have found that the NEAT unfiltered magnitudes track V-band SN lightcurves quite well. 


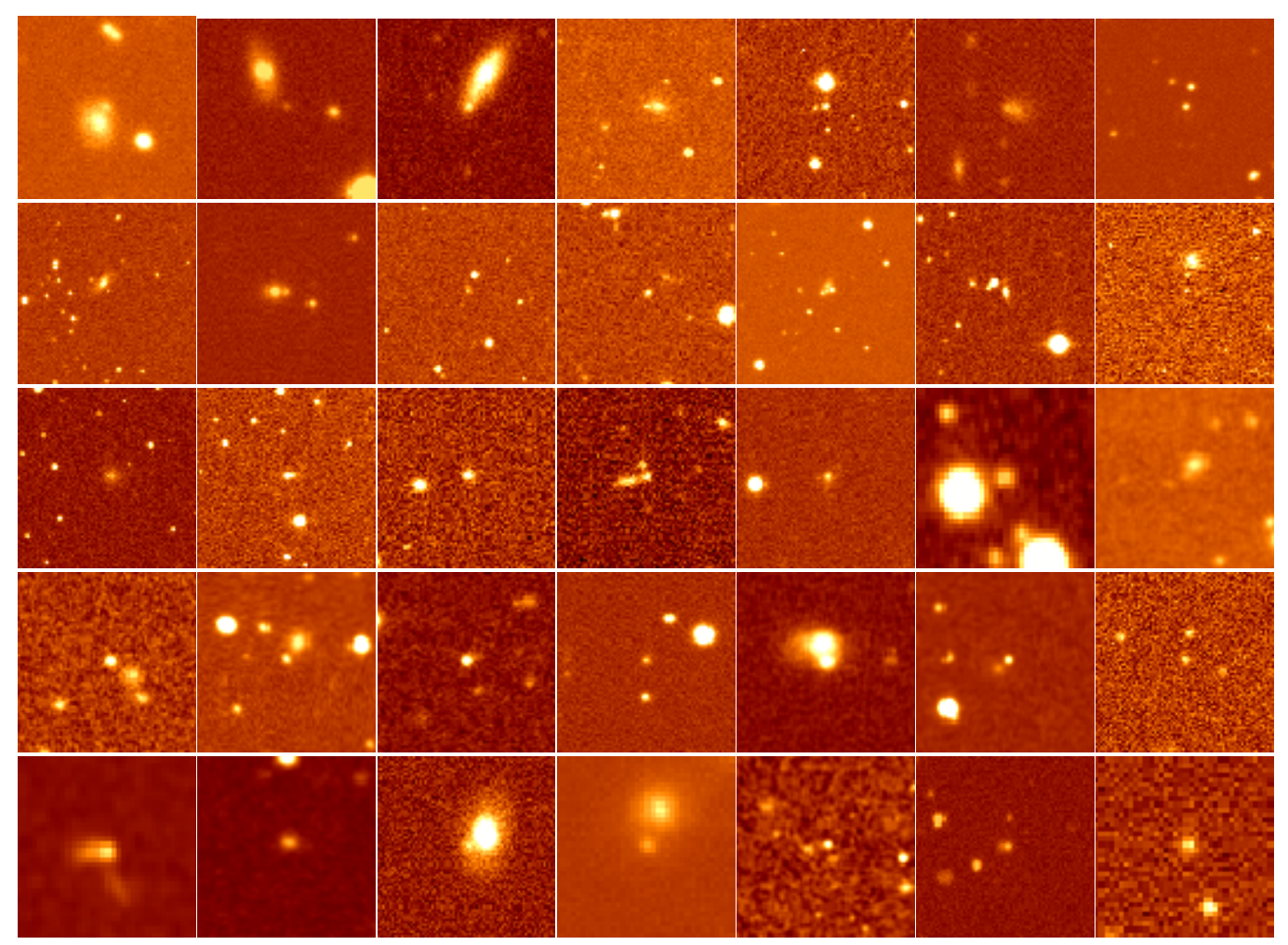

Fig. 1. A mosaic of the 35 supernovae found by the Nearby Supernova Factory pipeline in 2002. Each image is centered on the respective supernova.

telescope: $C_{\max }=S_{O} /\left(S_{D}-\frac{S_{0}}{365 \text { days }}\right)$. For the NEAT observation program, this cadence is approximately 20 days. An important goal of the SNfactory is to discover SNe early enough after explosion to follow them through their rise and fall. Fig. 3 shows a comparison of our simulations of the SNe discovery phase with the actual discovery phase for SNe from the SNfactory search having well-known dates of maximum. For shorter sky coverage cadences less sky can be covered and so fewer SNe are discovered overall, although the epoch of each SN can be better constrained. Fig. 3 clearly shows that the SNfactory search pipeline is successfully finding SNe Ia early in their lightcurves.

\section{Conclusion}

The Nearby Supernova Factory search pipeline is operational and has proven the ability to discover $\sim 12 \mathrm{SNe} /$ month $\Rightarrow \sim 150 \mathrm{SNe} /$ year. As $\frac{2}{3}$ of the supernovae discovered in our search have been $\mathrm{SNe}$ Ia, we expect to discover $\sim 100$ SNe Ia/year. Most of these supernovae have been discovered sufficiently early to enable detailed study starting before maximum light. This extensive study will enable improvements in the use of SNe Ia for cosmological measurements, and provide a wealth of information on the supernovae themselves. 


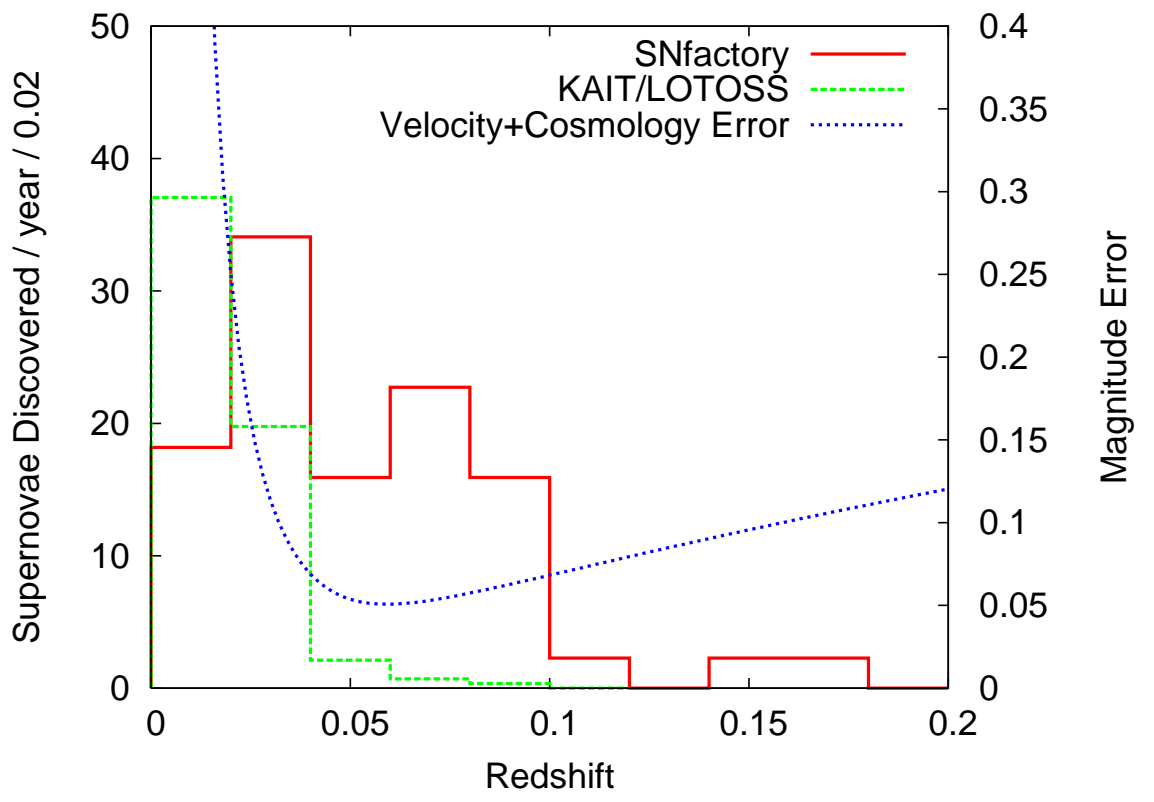

Fig. 2. The SNfactory is operating in the "sweet spot" redshift range between peculiar-velocity noise and cosmological uncertainty. The SNfactory curve is the redshift distribution of supernovae found and spectroscopically confirmed in our search to date scaled up to $100 \mathrm{SNe} /$ year. The velocity error is for an assumed $300 \mathrm{~km} / \mathrm{s}$ velocity dispersion. The cosmology error is modeled as the difference between an Einstein-de Sitter cosmology and a Universe with $\Omega_{M}=0.3$ and $\Omega_{\Lambda}=0.7$.

\section{Expected phases of $\mathrm{SNe}$}

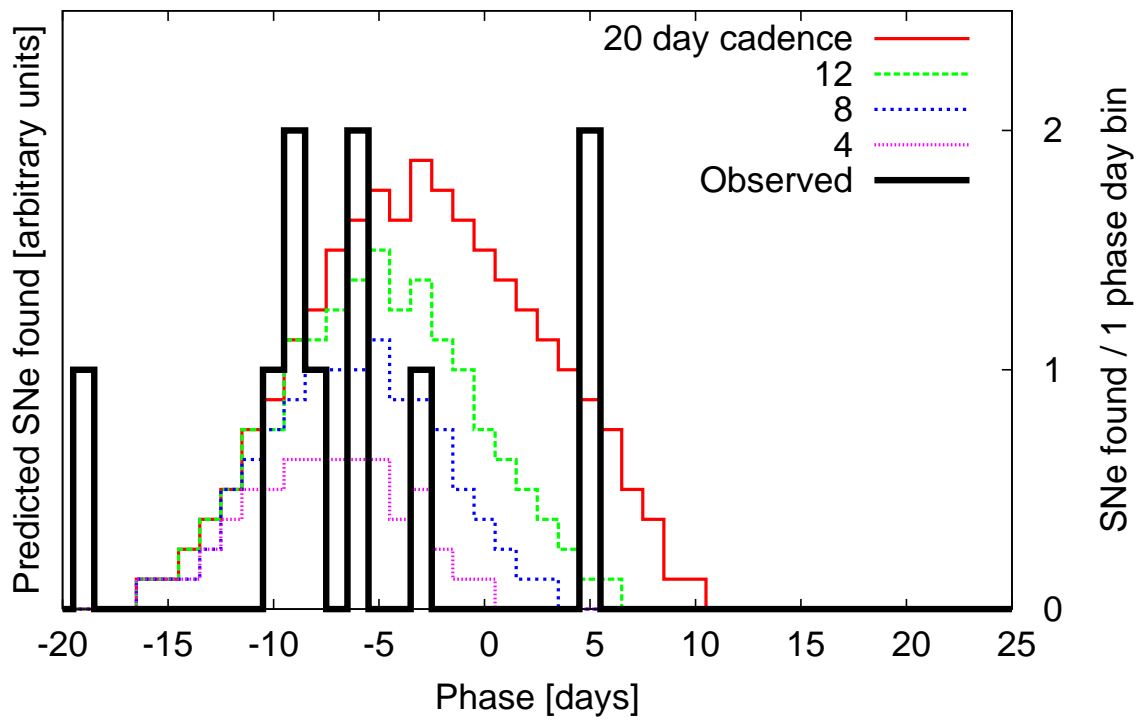

Fig. 3. The distribution of discovery epoch for the SNe Ia with determined dates of maximum found in the SNfactory dataset compared with the simulations described in the text. The model curves are not calculated in absolute units and have been scaled for comparison with the observations. As the model curves show, a shorter cadence gives fewer supernovae but better constraints on the epoch at discovery. 


\section{References}

Aldering, G. et al. 2002b, Ed. Dressler, A. M. Proc. SPIE, 4835, pp. 146-157

Aldering, G. et al. 2002a, Ed. Tyson, J. A.Proc. SPIE, 4836, pp. 61-72

Braun, H.-W. 2003, High Performance Wireless Research and Education Network http://hpwren.ucsd.edu/

Cabanela, J. E. et al. 2003, PASP, 115, 837

Garnavich, P. M. et al. 2002, BAAS, 34, 1233

Garnavich, P. M. et al. 1998, ApJ, 509, 74

Goldhaber, G. et al. 2001, ApJ, 558, 359

IAU. 2003, Minor Planet Checker http://scully.harvard.edu/ ${ }^{\sim}$ cgi/CheckMP

Lantz, B. et al. 2003, in Proc. SPIE, in press

Pécontal, E. et al. 2003, Proc. of the ESO/MPA/MPE July 2002, p. 404.

Pain, R. et al. 2003. Proc. ESO/MPA/MPE July 2002, p. 408.

Perlmutter, S. et al. 1997, ApJ, 483, 565

Perlmutter, S. et al. 1998, Nature, 391, 51

Perlmutter, S. et al. 1999, ApJ, 517, 565

Riess, A. G. et al. 1998, AJ, 116, 1009

Pravdo, S. H. et al. 1999, AJ, 117, 1616

The QUEST Collaboration. 2003, The Palomar-QUEST Variability Survey http://hepwww.physics.yale.edu/quest/palomar.html 